\title{
Bisection Method for Three-parameter Eigenvalue Problems
}

\author{
Songita Boruah \\ Research Scholar \\ Dept.of Mathematics \\ Dibrugarh University \\ Dibrugarh, Assam
}

\author{
Arun Kumar Baruah \\ Professor \\ Dept.of Mathematics \\ Dibrugarh University \\ Dibrugarh Assam
}

\begin{abstract}
This paper discusses the Bisection method for Threeparameter eigenvalue problems keeping one parameter constant. Finally some numerical results are presented to illustrate the performance and application of this method.
\end{abstract}

\section{Keywords}

Multiparameter, eigenvalue, eigenvector, Bisection Method

\section{INTRODUCTION}

Multiparameter eigenvalue problems are generalization of one-parameter eigenvalue problems and can be found when the method of separation of variables is applied to certain boundary value problems associated with partial differential equations. Much more works have been done in the field of one-parameter eigenvalue problems, both theoretically and numerically compared to two-parameter or more than twoparameter eigenvalue problems. Some works have been done theoretically in the field of multiparameter eigenvalue problems [11]. Few authors have dealt with the multiparameter eigenvalue problems numerically mainly in two-parametic cases. Numerical methods applied to a threeparameter problems are very limited and hence some contribution in this area are always in needed.

\section{THREE-PARAMETER EIGENVALUE PROBLEM AND ITS REDUCTION TO A SYSTEM OF ONE- PARAMETER PROBLEMS}

Consider the three-parameter eigenvalue problems

$A_{10} x=\lambda_{1} A_{11} x+\lambda_{2} A_{12} x+\lambda_{3} A_{13} x$

$A_{20} y=\lambda_{1} A_{21} y+\lambda_{2} A_{22} y+\lambda_{3} A_{23} y$

$A_{30} z=\lambda_{1} A_{31} z+\lambda_{2} A_{32} z+\lambda_{3} A_{33} z$

Where $\lambda_{i} \in \square, \mathrm{i}=1,2,3$ and

$$
\begin{aligned}
& x \in \square^{n} \backslash\{0\}, \mathrm{A}_{10}, \mathrm{~A}_{11}, \mathrm{~A}_{12}, \mathrm{~A}_{13} \in \square^{n \times n} \\
& y \in \square^{m} \backslash\{0\}, \mathrm{A}_{20}, \mathrm{~A}_{21}, \mathrm{~A}_{22}, \mathrm{~A}_{23} \in \square^{m \times m} \\
& z \in \square^{p} \backslash\{0\}, \mathrm{A}_{30}, \mathrm{~A}_{31}, \mathrm{~A}_{32}, \mathrm{~A}_{33} \in \square^{p \times p}
\end{aligned}
$$

Where $\lambda_{i} \in \square, \mathrm{i}=1,2,3$ are called the eigenvalues and $x, y, z$ are called eigenvectors of the problem.

Problem (1.2.1) can be reduced to a system of three oneparameter problems:

$$
\begin{aligned}
& \Delta_{1} u=\lambda_{1} \Delta_{0} u \\
& \Delta_{2} u=\lambda_{2} \Delta_{0} u \\
& \Delta_{3} u=\lambda_{3} \Delta_{0} u
\end{aligned}
$$

where $\Delta_{0}, \Delta_{1}, \Delta_{2}, \Delta_{3}$ are $(\mathrm{mnp}) \times(\mathrm{mnp})$ dimensional matrices defined as

$$
\begin{aligned}
\Delta_{0}= & A_{11} \otimes A_{22} \otimes A_{33}-A_{11} \otimes A_{23} \otimes A_{32} \\
+ & A_{12} \otimes A_{23} \otimes A_{31}-A_{12} \otimes A_{21} \otimes A_{33} \\
& +A_{13} \otimes A_{21} \otimes A_{32}-A_{13} \otimes A_{22} \otimes A_{31} \\
\Delta_{1}= & A_{10} \otimes A_{22} \otimes A_{33}-A_{10} \otimes A_{23} \otimes A_{32}+A_{12} \otimes A_{23} \otimes A_{30}-A_{12} \otimes A_{20} \otimes A_{33} \\
& \quad(1.2 .4) \\
& +A_{13} \otimes A_{20} \otimes A_{32}-A_{13} \otimes A_{22} \otimes A_{30} \\
\Delta_{2}= & A_{11} \otimes A_{20} \otimes A_{33}-A_{11} \otimes A_{23} \otimes A_{30}+A_{10} \otimes A_{23} \otimes A_{31}-A_{10} \otimes A_{21} \otimes A_{33} \\
& +A_{13} \otimes A_{21} \otimes A_{30}-A_{13} \otimes A_{20} \otimes A_{31} \\
\Delta_{3}= & A_{11} \otimes A_{22} \otimes A_{30}-A_{11} \otimes A_{20} \otimes A_{32}+A_{12} \otimes A_{20} \otimes A_{31}-A_{12} \otimes A_{21} \otimes A_{30} \\
& +A_{10} \otimes A_{21} \otimes A_{32}-A_{10} \otimes A_{22} \otimes A_{31}
\end{aligned}
$$

And

$u=x \otimes y \otimes z$

Theorem : Let $\left(\lambda_{1}, \lambda_{2}, \lambda_{3}\right)$ be an eigenvalue and $(\mathrm{x}, \mathrm{y}, \mathrm{z})$ a corresponding eigenvector of the system (1.2.1) then $\left(\lambda_{1}, \lambda_{2}, \lambda_{3}\right)$ is an eigenvalue of the system (1.2.2) and $u=x \otimes y \otimes z$ is the corresponding eigenvector.

Definition 1.3.1. The Kronecker product (.®.): $\square^{m \times n} \times \square^{p \times q} \rightarrow \square^{m p \times n q}$ is defined by

$A \otimes B=\left[\begin{array}{ccc}a_{11} B & \cdots & a_{1 n} B \\ \vdots & \ddots & \vdots \\ a_{n 1} B & \cdots & a_{n n} B\end{array}\right]$

Where we use the standard notation $(A)_{i j}=a_{i j}$

The Kronecker product is a special case of the tensor product, 
and as such it inherits the properties of bilinearity and associativity, i.e.

$$
\begin{aligned}
& (\mathrm{kA}) \otimes \mathrm{B}=\mathrm{A} \otimes(\mathrm{kB})=\mathrm{k}(\mathrm{A} \otimes \mathrm{B}) \\
& \mathrm{A} \otimes(\mathrm{B}+\mathrm{C})=\mathrm{A} \otimes \mathrm{B}+\mathrm{A} \otimes C \\
& (\mathrm{~A}+\mathrm{B}) \otimes \mathrm{C}=\mathrm{A} \otimes C+B \otimes C
\end{aligned}
$$

We now establish a famous property of the Kronecker product, from [9].

\section{BISECTION METHOD}

The main objective of this paper is to use the bisection method for the following three-parameter eigenvalue problem

$$
\begin{aligned}
&\left(A_{10}-\lambda_{1} A_{11}-\lambda_{2} A_{12}-\lambda_{3} A_{13}\right) X=0 \\
&\left(A_{20}-\lambda_{1} A_{21}-\lambda_{2} A_{22}-\lambda_{3} A_{23}\right) Y=0 \\
&\left(A_{30}-\lambda_{1} A_{31}-\lambda_{2} A_{32}-\lambda_{3} A_{33}\right) Z=0
\end{aligned}
$$

Where $A_{i 0} \in R^{n \times n}$ are irreducible symmetric tridiagonal matrices, $A_{i j}(i=1,2, \ldots, n, j=1,2, \ldots, n)$ are non-singular diagonal matrices. $\left(\lambda_{1}, \lambda_{2}, \lambda_{3}\right)$ and $X, Y, Z$ are eigenvalues and corresponding eigenvectors of the three-parameter eigenvalue problems (2.11).

In this paper one parameter (say $\lambda_{3}$ ) is considered as fix and then Bisection Method is applied for Three-parameter eigenvalue problem. To estimate this fix value we apply Gerschgorin Theorem. The Theorem states that all the eigenvalues of a matrix $[A]_{n \times n}$ lie in the union of the Disks $D_{i}$ for $\mathrm{i}=1,2, \ldots, \mathrm{n}$.

Where $D_{i}=\left\{z \in C|| z-A_{i i} \mid \leq r_{i}\right\}, r_{i}=\sum_{j \neq i}\left|A_{i j}\right|$.

Using this Gerschgorin Theorem for the third equation of (1.2.2) an approximate value for $\lambda_{3}$ is obtained. Then the three-parameter eigenvalue problem (2.1.1) reduces to the following two parameter eigenvalue problem

$$
\begin{aligned}
\left(W_{1}-\lambda_{1} A_{11}-\lambda_{2} A_{12}\right) X & =0 \\
\left(W_{2}-\lambda_{1} A_{21}-\lambda_{2} A_{22}\right) Y & =0 \\
\left(W_{3}-\lambda_{1} A_{31}-\lambda_{2} A_{32}\right) Z & =0
\end{aligned}
$$

Where $W_{i}=A_{i 0}+\lambda_{3} A_{i 3}$

Consider $A_{1}\left(\lambda_{1}, \lambda_{2}\right)=\operatorname{det}\left(W_{1}-\lambda_{1} A_{11}-\lambda_{2} A_{12}\right)$

$A_{2}\left(\lambda_{1}, \lambda_{2}\right)=\operatorname{det}\left(W_{2}-\lambda_{1} A_{21}-\lambda_{2} A_{22}\right)$

$A_{3}\left(\lambda_{1}, \lambda_{2}\right)=\operatorname{det}\left(W_{3}-\lambda_{1} A_{31}-\lambda_{2} A_{32}\right)$

The aim of this paper is to find the eigen pair $\left(\lambda_{1}, \lambda_{2}\right)$. So one has to find the intersection points of $A_{1}\left(\lambda_{1}, \lambda_{2}\right), A_{1}\left(\lambda_{1}, \lambda_{2}\right)$, $A_{1}\left(\lambda_{1}, \lambda_{2}\right)$ in $\left(\lambda_{1}, \lambda_{2}\right)$ plane.

Let us denote $f_{i k}\left(\lambda_{1}, \lambda_{2}\right)$ as the leading principal minors of $W_{i}-\lambda_{1} A_{i 1}-\lambda_{2} A_{i 2}$. Now applying Gerschgorin Theorem to estimate rough bounds of $\lambda_{1}, \lambda_{2}$. Now from these rough bounds of $\lambda_{1}, \lambda_{2}$ we consider a rectangle $\left[a_{1}, a_{2} ; b_{1}, b_{2}\right]$ where the approximate eigenvalues lie. If $\left(\lambda_{1}, \lambda_{2}\right)$ lies on the rectangle $\left[a_{1}, a_{2} ; b_{1}, b_{2}\right]$ then there must be eigencurves of $f_{1}=0, f_{2}=0, f_{3}=0$ that cross through at least one of any four sides of the rectangle.
First consider any two equations say (2.1.3) and (2.1.4) and apply the following algorithm for two dimensional Bisection Method

(1) Calculate respectively $\left.\left.f_{1, j}\right\}_{0}^{n}, \quad f_{2, j}\right\}_{0}^{n}$ and then calculate the number of sign changes at the vertices of $\lambda_{1}=a_{1}, a_{2}$ and $\lambda_{2}=b_{1}, b_{2}$ and form the differences between the two corresponding numbers for each side.

Let $V_{11}, V_{12}, V_{13}, V_{14}$ and $V_{21}, V_{22}, V_{23}, V_{24}$ denote the absolute values of these differences for $\left.f_{1, j}\right\}_{0}^{n}$, $\left.f_{2, j}\right\}_{0}^{n}$ respectively and set $V_{1}=V_{11}+V_{12}+V_{13}+$ $V_{14}$ and

$$
V_{2}=V_{21}+V_{22}+V_{23}+V_{24}
$$

(2) If $V_{1} * V_{2}=0$, then there is no solution in $\left[a_{1}, a_{2} ; b_{1}, b_{2}\right]$

(3) If $V_{1} * V_{2} \neq 0$

(i) If $\max \left\{\left|a_{1}-a_{2}\right|,\left|b_{1}-b_{2}\right|\right\} \leq \epsilon$ suitable criterion for termination ) then we have an approximate eigenvalue pair $\lambda_{1}=\frac{\left(a_{1}+a_{2}\right)}{2}, \lambda_{2}=\frac{\left(b_{1}+b_{2}\right)}{2}$ othewise

(ii) Taking $a_{3}=\frac{\left(a_{1}+a_{2}\right)}{2}, \quad b_{3}=\frac{\left(b_{1}+b_{2}\right)}{2}$ we obtain four smaller rectangles

(4) Repeat the above step (1)-(3) for each smaller rectangle

Again we consider equation (2.1.4) and (2.1.5) and applying the above algorithm. The eigenvalues those are common for both cases will give us the approximate eigenvalues.

\subsection{Numerical Result:}

Consider the three-parameter eigenvalue problems

$\left(\begin{array}{ll}1 & 2 \\ 3 & 4\end{array}\right)\left(\begin{array}{l}x_{1} \\ x_{2}\end{array}\right)=$

$\lambda_{1}\left(\begin{array}{ll}2 & 0 \\ 0 & 3\end{array}\right)\left(\begin{array}{l}x_{1} \\ x_{2}\end{array}\right)+\lambda_{2}\left(\begin{array}{ll}1 & 0 \\ 0 & 1\end{array}\right)\left(\begin{array}{l}x_{1} \\ x_{2}\end{array}\right)+$

$\lambda_{3}\left(\begin{array}{ll}4 & 0 \\ 0 & 5\end{array}\right)\left(\begin{array}{l}x_{1} \\ x_{2}\end{array}\right)$

(3.1.1)

$\left(\begin{array}{ll}2 & 5 \\ 4 & 1\end{array}\right)\left(\begin{array}{l}y_{1} \\ y_{2}\end{array}\right)=$

$\lambda_{1}\left(\begin{array}{ll}1 & 0 \\ 0 & 4\end{array}\right)\left(\begin{array}{l}y_{1} \\ y_{2}\end{array}\right)+\lambda_{2}\left(\begin{array}{ll}3 & 0 \\ 0 & 4\end{array}\right)\left(\begin{array}{l}y_{1} \\ y_{2}\end{array}\right)+$

$\lambda_{3}\left(\begin{array}{ll}6 & 0 \\ 0 & 5\end{array}\right)\left(\begin{array}{l}y_{1} \\ y_{2}\end{array}\right)$

$$
\begin{gathered}
\left(\begin{array}{ll}
3 & 2 \\
1 & 7
\end{array}\right)\left(\begin{array}{l}
z_{1} \\
z_{2}
\end{array}\right)=\lambda_{1}\left(\begin{array}{ll}
5 & 0 \\
0 & 7
\end{array}\right)\left(\begin{array}{l}
z_{1} \\
z_{2}
\end{array}\right)+\lambda_{2}\left(\begin{array}{ll}
3 & 0 \\
0 & 2
\end{array}\right)\left(\begin{array}{l}
z_{1} \\
z_{2}
\end{array}\right) \\
+\lambda_{3}\left(\begin{array}{ll}
1 & 0 \\
0 & 8
\end{array}\right)\left(\begin{array}{l}
z_{1} \\
z_{2}
\end{array}\right)
\end{gathered}
$$

Reducing the three-parameter eigenvalue problem to a system of three one parameter eigenvalue problem using kronecker product method we apply Geroschgorin Theorem for estimating an approximate value of $\lambda_{3}$. consider $\lambda_{3}=.0290$. Putting $\lambda_{3}=.0290$ the given three-parameter eigenvalue problem becomes

$\left(\begin{array}{cc}8840 & 2 \\ 3 & 3.8550\end{array}\right)\left(\begin{array}{l}x_{1} \\ x_{2}\end{array}\right)=\lambda_{1}\left(\begin{array}{ll}2 & 0 \\ 0 & 3\end{array}\right)\left(\begin{array}{l}y_{1} \\ y_{2}\end{array}\right)+\lambda_{2}\left(\begin{array}{ll}1 & 0 \\ 0 & 1\end{array}\right)\left(\begin{array}{l}x_{1} \\ x_{2}\end{array}\right)$
$\left(\begin{array}{ll}3.1 .2\end{array}\right)$
$\left(\begin{array}{cc}1.8260 & 5 \\ 4 & .8550\end{array}\right)\left(\begin{array}{l}y_{1} \\ y_{2}\end{array}\right)=\lambda_{1}\left(\begin{array}{ll}1 & 0 \\ 0 & 4\end{array}\right)\left(\begin{array}{l}y_{1} \\ y_{2}\end{array}\right)+\lambda_{2}\left(\begin{array}{ll}3 & 0 \\ 0 & 4\end{array}\right)\left(\begin{array}{l}y_{1} \\ y_{2}\end{array}\right)$
$\left(\begin{array}{l}3.1 .3\end{array}\right)$




$$
\left(\begin{array}{cc}
2.9710 & 2 \\
1 & 6.7680
\end{array}\right)\left(\begin{array}{l}
z_{1} \\
z_{2}
\end{array}\right)=\lambda_{1}\left(\begin{array}{ll}
5 & 0 \\
0 & 7
\end{array}\right)\left(\begin{array}{l}
z_{1} \\
z_{2}
\end{array}\right)+\lambda_{2}\left(\begin{array}{ll}
3 & 0 \\
0 & 2
\end{array}\right)\left(\begin{array}{l}
z_{1} \\
z_{2}
\end{array}\right)
$$

Consider the approximate rectangle is form by the points $(0,-$ 6.9786), (0,6.9786), (5.4782,0), (-5.4782,0). Now applying bisection algorithm for (3.1.2), (3.1.3) and (3.1.3), (3.1.4) separately will give the approximate eivgenvalues. Some approximate values are

$$
\begin{aligned}
& \left(\lambda_{1}, \lambda_{2}\right)=(3.1028,-.4088) \\
& \left(\lambda_{1}, \lambda_{2}\right)=(4.5377, .0545) \\
& \left(\lambda_{1}, \lambda_{2}\right)=(2.6963,3.4348) \\
& \left(\lambda_{1}, \lambda_{2}\right)=(-.0428, .0545)
\end{aligned}
$$

\section{CONCLUSIONS}

In this paper Bisection method for three-parameter eigenvalue problem is not used directly. In this paper two-dimensional Bisection method for three-parameter eigenvalue problem is used considering one parameter fix one-parameter fix. In this method the rate of convergence is slow but one can use this method very easily to find starting approximation for $\left(\lambda_{1}, \lambda_{2}, \lambda_{3}\right)$.

\section{REFERENCES}

[1] Atkinson, F.V., 1972. 'Multiparameter Eigenvalue Problems', (Matrices and compact operators) Academic Press, New York, Vol.1

[2] Atkinson, F.V., 1968. 'Multiparameter spectral theory', Bull.Am.Math.Soc., Vol.75, pp(1-28)

[3] Baruah, A.K., 1987. 'Estimation of eigen elements in a two-parameter eigen value problem', Ph.D Thesis, Dibrugarh University, Assam.

[4] Binding, P and Browne P. J., (1989). 'Two parameter eigenvalue problems for matrices', Linear algebra and its application, $p p(139-157)$
[5] Browne, P.J., 1972. 'A multiparameter eigenvalue problem'. J. Math. Analy. And Appl. Vol. 38, pp(553568)

[6] Changmai, J., 2009. 'Study of two-parameter eigenvalue problem in the light of finite element procedure'. Ph. D Thesis, Dibrugarh University, Assam.

[7] Collatz, L.(1968). 'Multiparameter eigenvalue problems in linear product spaces', J. Compu. and Syst.Scie., Vol. 2, $p p(333-341)$

[8] Fox, L., Hayes, L. And Mayers, D.F., 1981. 'The double eigenvalue problems, Topic in Numerical Analysis ', Proc. Roy. Irish Acad. Con., Univ. College, Dublin, 1972, Academic Press, pp(93-112)

[9] Horn, R.A,1994. ' Topics in Matrix Analysis'. Cambridge, Cambridge University.

[10] Hua Dai , 2007. "Numerical methods for solving multiparameter eigenvalue problems," International Journal of Computer Mathematics, 72:3, 331-347

[11] Konwar, J., 2002. 'Certain studies of two-parameter eigenvalue problems', Ph.D Thesis, Dibrugarh University, Assam.

[12] Browne Philip A. 'Numerical Methods for Twoparameter eigenvalue problem'.

[13] Plestenjak, B., 2003. Lecture Slides, ' Numerical methods for algebraic two parameter eigenvalue problems', Ljubljana, University of Ljubljans.

[14] Roach, G.F., (1976). 'A Fredholm theory for multiparameter problems', Nieuw Arch. V. Wiskunde, Vol.XXIV(3), pp(49-76)

[15] Sleemen, B. D., 1971. 'Multiparameter eigenvalue problem in ordinary differential equation'. Bul. Inst. Poll. Jassi. Vol. 17, No. 21 pp(51-60)

[16] Sleeman, B.D., 1978, "Multiparameter Spectral Theory in Hilbert Space," Pitman Press, London 